\title{
Wind-induced Nonlinearities of Super Long Stay Cables
}

\author{
Mingjie Zhang ${ }^{1, ~ a, ~ F u y o u ~} \mathrm{Xu}^{1, b}$ \\ ${ }^{1}$ Institute of Bridge Engineering, Dalian University of Technology, Dalian, Liaoning, China \\ amingjiezhg@hotmail.com, bfuyouxu@dlut.edu.cn
}

Keywords: Stay cable; Effective elastic modulus; Fundamental frequency; Parametric vibration; Aerostatic loading.

Abstract. Effects of aerostatic wind loads on effective elastic modulus and fundamental frequencies of stay cables in bridge are studied by finite element method (FEM). Dynamic responses of stay cables under supports' excitations are calculated through theoretical method and FEM. Results show that the in-plane fundamental frequency of a cable is higher than the corresponding out-plane frequency. With external excitations, stay cables can be excited to exhibit large amplitude vibration. Parametric vibrations of cables are not perfectly symmetrical about the equilibrium curves. Under the combined supports' excitation and aerostatic wind action, a stay cable will vibrate around its deformed curve under self weight and aerostatic load.

\section{Introduction}

Cable-stayed bridges have undergone rapid developments worldwide, leading to notable progresses. Nowadays, the first three longest cable-stayed bridges are Russky Island Bridge in Russia (1104 m), Sutong Bridge $(1088 \mathrm{~m})$ and Stonecutters Bridge in China $(1018 \mathrm{~m})$, respectively. The longest stay cables of these bridges are approaching $600 \mathrm{~m}$, which are susceptible to large amplitude vibration under external excitations. Cable vibration can be classified into vortex-induced vibration, wake galloping, rain-wind induced vibration, and parametric vibration, etc.

Effective elastic modulus (EEM) and fundamental frequency (FF) of a stay cable are important parameters reflecting its nonlinear and dynamic characteristics. In the present paper, the effects of aerostatic loads on the effective elastic modulus and fundamental frequency of four cables from Sutong Bridge are studied, as well as the dynamic behaviors of cables induced by parametric excitations. Both theoretical method and finite element method are used in this paper.

\section{Basic Information of Stay Cables}

Basic information of the four stay cables studied in the present paper are listed in Table. 1.

Table 1 Basic information of stay cables

\begin{tabular}{c|cccc}
\hline Norameters & $\mathrm{J} 12$ & $\mathrm{~J} 19$ & $\mathrm{~J} 26$ & $\mathrm{~J} 34$ \\
\hline Length/[m] & 251.2 & 349.5 & 454.6 & 578.6 \\
\hline Inclined angle/[deg.] & 43.89 & 33.03 & 26.86 & 22.65 \\
\hline Area/[cm $\left.{ }^{2}\right]$ & 62.73 & 81.2 & 92.75 & 120.46 \\
\hline Initial tension/[kN] & 3482 & 4336 & 5015 & 6708 \\
\hline Mass/[kg/m] & 53.2 & 68.6 & 77.7 & 100.8 \\
\hline Elastic modulus/[GPa] & 190 & 190 & 190 & 190 \\
\hline Elevation/[m] & $(76.3,249.4)$ & $(77.2,267.0)$ & $(77.8,282.4)$ & $(78.0,300.0)$ \\
\hline
\end{tabular}

The lengths denote the straight line distances between the two anchoring points on the deck and tower; the elevations are the altitudes of two anchoring points. Moreover, supports' excitations are along the straight line connecting the two anchoring points, and defined to be positive in the tensioning direction. 


\section{Effective Elastic Modulus (EEM) and Fundamental Frequency (FF) of Stay Cables}

Finite element analyses are carried out using ANSYS program. The link 10 unit is used to model the stay cables with one meter interval. Large deformation effect and stress stiffening effect are considered in the finite element analyses.

EEM and FF of stay cables in wind flows with basic wind speeds of $0 \mathrm{~m} / \mathrm{s}, 50 \mathrm{~m} / \mathrm{s}$, and $100 \mathrm{~m} / \mathrm{s}$ are calculated. The wind direction is supposed to be perpendicular to the vertical plane, and the calculations are carried out with the following assumptions:

(1) The two anchoring points keep fixed under self weight and static wind load;

(2) Wind speed distribution along the vertical height follows the power law index model;

(3) Drag coefficient of stay cables is 0.7 and the ground surface roughness coefficient is 0.12 .

The calculated EEM of stay cables in wind flows are listed in Table 2. It shows that all EEM of the cables are lower than 190GPa that at $0 \mathrm{~m} / \mathrm{s}$, which is caused by the sag effect under self weights. As wind speed increases, the lateral displacements caused by wind effects also result in nonlinear behavior of cables. The EEM of cables are further decreased.

Table 2 EEM of stay cables at different wind speeds

\begin{tabular}{cc|ccc}
\hline NO. & wind speed[m/s] & 0 & 50 & 100 \\
\hline J12 & 186 & 185 & 167 \\
\hline J19 & 180 & 178 & 156 \\
\hline J26 & 173 & 171 & 148 \\
\hline J34 & 167 & 164 & 143 \\
\hline
\end{tabular}

The FF of cables in wind flows are listed in Table. 3. It shows that the out-plane FF is lower than the in-plane FF, and the deviation increases with wind speed. Both out-plane FF and in-plane FF increase with wind speed.

Table 3 FF of stay cables at different wind speeds

\begin{tabular}{|c|c|c|c|c|}
\hline \multicolumn{2}{|c|}{$\begin{array}{l}\text { wind } \text { speed }[\mathrm{m} / \mathrm{s}] \\
\text { NO. }\end{array}$} & 0 & 50 & 100 \\
\hline \multirow{2}{*}{$\mathrm{J} 12$} & out-plane & 0.5124 & 0.5135 & 0.5275 \\
\hline & in-plane & 0.5175 & 0.5206 & 0.5607 \\
\hline \multirow{2}{*}{ J19 } & out-plane & 0.3653 & 0.3665 & 0.3813 \\
\hline & in-plane & 0.3752 & 0.3786 & 0.4195 \\
\hline \multirow{2}{*}{ J26 } & out-plane & 0.2865 & 0.2876 & 0.3018 \\
\hline & in-plane & 0.2996 & 0.3029 & 0.3410 \\
\hline \multirow{2}{*}{$\mathrm{J} 34$} & out-plane & 0.2314 & 0.2325 & 0.2442 \\
\hline & in-plane & 0.2468 & 0.2497 & 0.2806 \\
\hline
\end{tabular}

Under the self weight and aerostatic wind load actions, the vertical and lateral deformations of stay cables are generated, which change the distribution of internal force of stay cables. Therefore, the stiffness and frequencies of in-plane and out-plane vibration are different. Further studies indicate that the out-plane FF is lower than the in-plane FF for symmetrical modes of vibration while the out-plane FF is higher than the in-plane FF for asymmetrical modes of vibration. For the in-plane vibration of a symmetrical mode, the deformation (under self weight and static wind load) and vibration displacement are in the same plane. The equilibrium curve of the vibration is the deformed curve of the stay cable. The internal force of the stay cable is increased after deformation, so the stiffness for in-plane vibration of the cable is higher and the in-plane frequency is higher. Such stiffening effect is less significant for the out-plane vibration and the out-plane frequency is lower. On the contrary, for the vibrations of asymmetrical modes, the cable has two or more sections vibrating around the equilibrium curve in two opposite directions, and the stiffening effect of deformation is then weakened. The out-plane vibration is in perpendicular with deformation and the effect of deformation on out-plane vibration is not changed between the symmetrical and asymmetrical modes. So, the frequency of out-plane mode is higher than that of the in-plane one for 
the asymmetrical modes. The lateral deformation of a stay cable increases with wind speed. The difference between the out-plane and in-plane FF increases with wind speed.

\section{Responses of Stay Cables under Supports' Excitation}

Stay cables are prone to vibrate due to the direct loads (wind and/or rain) or excitations of supports (deck and/or tower). In recent years, cable vibrations induced by parametric excitations due to deck or tower motions were indeed observed in real bridges [1], and several studies have tried to address this problem. The conventional approach is to investigate the cable vibration given supports' motions of deck and/or tower. Though the model has some disadvantages, it can predict vibration of stay cables with acceptable accuracy.

A simplified model of a stay cable is shown in Fig. 1. The configuration of stay cable under self weight is supposed to be a parabolic curve in the $x-y$ plane. The excitation of deck is supposed to be sinusoidal and denoted as $U_{x}=U_{d} \sin \Omega t$. The tower is supposed to be static and rigid without loss of generality. The equation of vertical motion can be expressed as

$$
\frac{\partial}{\partial s}\left[(T+\tau)\left(\frac{d y}{d s}+\frac{\partial v}{\partial s}\right)\right] d s=m \frac{\partial^{2} v}{\partial t^{2}}-m g \cos \theta+c_{y} \frac{\partial v}{\partial t}
$$

where $T$ is the initial tension of the stay cable and $\tau$ is the variation of $T ; \theta$ is the inclined angle; $m$ is the mass of the cable per meter; $y$ is the configuration of the cable under self weight; $v$ is the vertical displacement of the cable relative to $y ; c_{y}$ is the viscous damping coefficient of vertical motion; $\Omega$ is the frequency of external excitation, and $\omega_{1}$ is the in-plane FF of the cable.

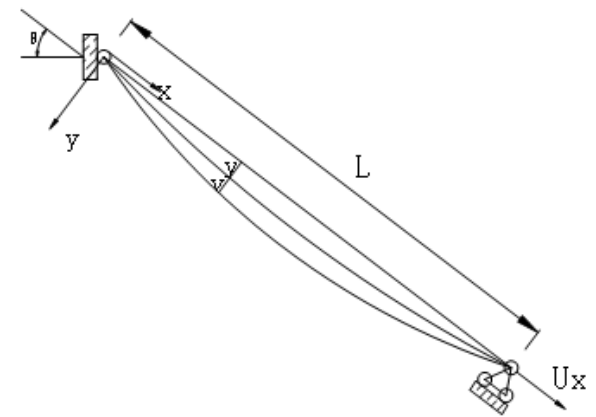

Fig. 1 Simplified model of a stay cable

The Mathieu differential equation is in agreement with the equation in the paper [2]:

$$
\alpha+2 \xi_{1} \omega_{1} \alpha+\left(\omega_{1}^{2}+\alpha_{1} \cos \Omega t\right) v_{1}+3 \alpha_{2} v_{1}^{2}+\alpha_{3} v_{1}^{3}+4 \alpha_{4} \cos \Omega t=0
$$

where, $\omega_{1}^{2}=\omega_{0}^{2}\left[1+\frac{1}{2}\left(\frac{2}{\pi}\right)^{4} \lambda^{2}\right], \omega_{0}^{2}=\pi^{2} H / m L^{2}, 2 \xi_{1} \omega_{1}=c_{y} / m, \alpha_{1}=\pi^{2} E A U_{d} / m L^{3}$

$\alpha_{2}=\pi E A g \cos \alpha / H L^{2}, \alpha_{3}=\pi^{4} E A / 4 m L^{4}, \alpha_{4}=E A g U_{d} \cos \alpha / \pi H L$

$d=m g \cos \alpha L^{2} / 8 H, L_{e}=L\left[1+8(d / L)^{2}\right], \lambda^{2}=[m g L \cos \alpha / H]^{2} /\left(H L_{e} / E A\right)$

The displacement responses of cable $J 34$ at $\xi=0.001, U_{d}=0.0001 L$ is calculated by Eq. (2) using the fourth-order Runge-Kutta method and is compared to the results from FEM. Responses of $J 34$ at $\Omega / \omega_{1}=1$ and $\Omega / \omega_{1}=2$ are shown in Fig. 2 and Fig. 3, respectively. The displacement is defined to be positive downward. It can be concluded that stay cables can be excited into large amplitude vibrations by extremely small excitations while the frequency ratio $\left(\Omega / \omega_{1}\right)$ is around 1 or 2 . Amplitudes in Fig. 2 and Fig. 3 are larger than $6 \mathrm{~m}$. Before reaching the steady state oscillation, the amplitude is constantly changing, showing a phenomenon of "flap". The results of Eq. (2) are in good agreement with that of FEM, indicating that the model provide good predictions of vibration.

It is worth noting that the vibration of the stay cable is not perfectly symmetrical about its equilibrium curve. The upward amplitude is larger than the downward amplitude. Further studies indicate that the asymmetry can be weakened by increasing the inclined angle (from 0 deg. to 90 deg.) and the vibration is perfectly symmetrical while the inclined angle is $90 \mathrm{deg}$. It could be 
inferred that the asymmetry in amplitudes is related to the sag of its equilibrium curve. The equilibrium curve of vibration is the deformed curve under self weight. When the cable moves downward or upward with a same displacement, the downward displacement produces a greater tension force. The tension generated by downward motion is larger than that of the upward motion of the same amplitude, which makes it more difficult for the cable to move downwards. Consequently, the upward amplitude is larger than the downward amplitude.

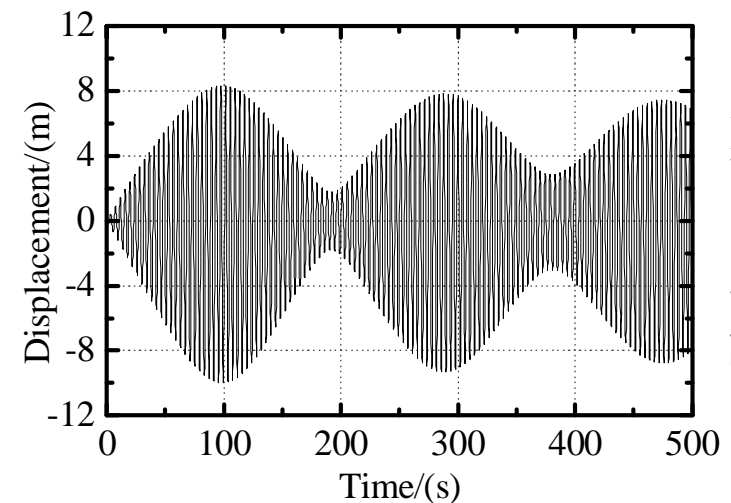

(a) Result of Eq. (2)

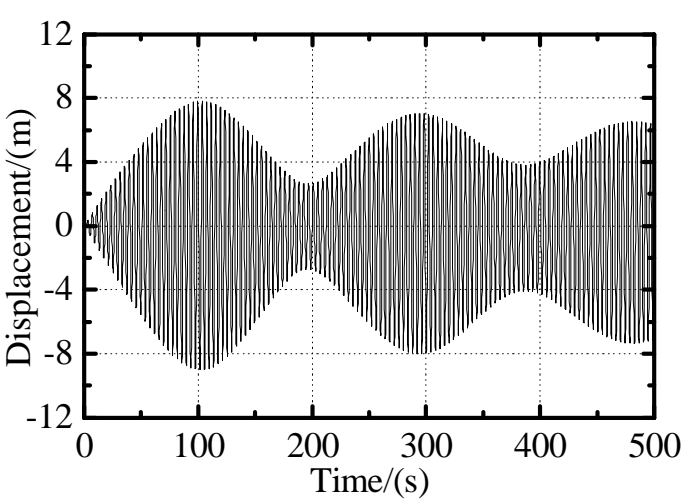

(b) Result of FEM

Fig. 2 Comparison of results of Eq. (2) and FEM at $\xi=0.001, \Omega / \omega_{I}=1$ and $U_{d}=0.0001 L$

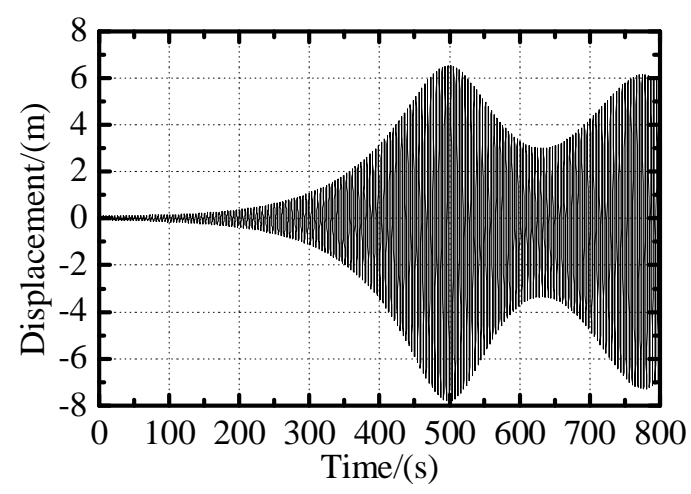

(a) Result of Eq. (2)

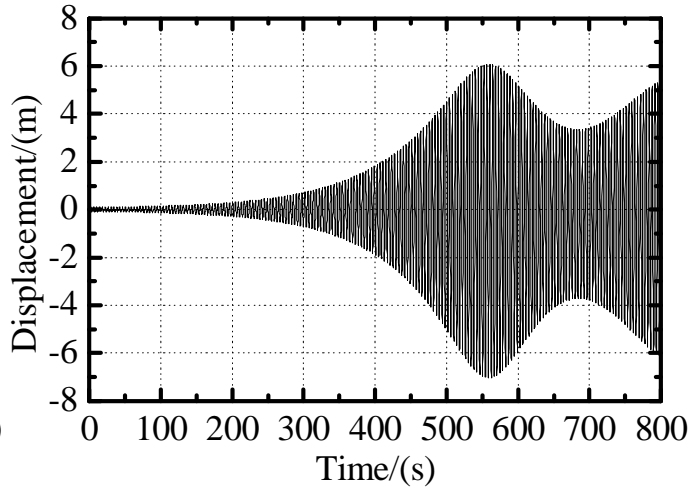

(b) Result of FEM

Fig. 3 Comparison of results of Eq. (2) and FEM at $\xi=0.001, \Omega / \omega_{1}=2$ and $U_{d}=0.0001 L$

To investigate the frequency ratio $\left(\Omega / \omega_{1}\right)$ range of large amplitude parametric vibration occurrence and the effects of external excitation strength on resultant vibration amplitudes, the dynamic responses of cable $J 34$ are calculated by Eq. (2) using Runge-Kutta method at $\xi=0.001$, $U_{d}=0.0001 L, 0.0002 L, 0.0003 L$, and the amplitudes are shown in Fig. 4 .

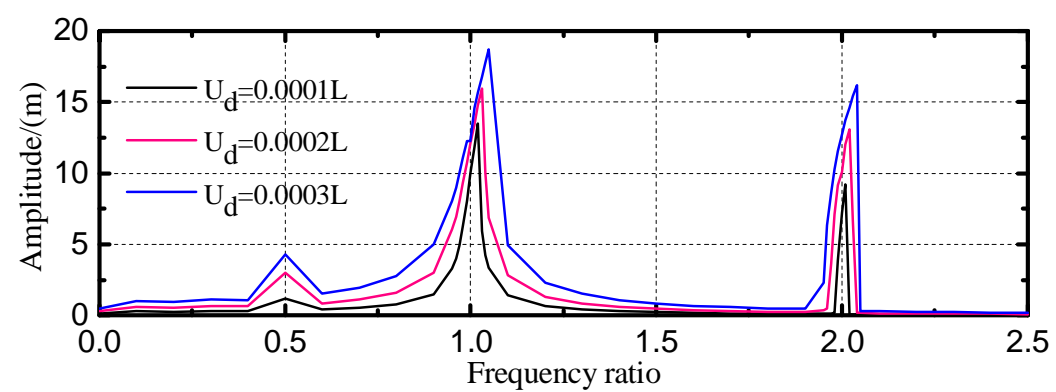

Fig. 4 Amplitude of displacement response of $J 34$ with respect to frequency ratio

It shows that there are three peaks around $0.5,1$ and 2, respectively. The peaks around 1 and 2 are not exactly at the positions of 1 and 2 but a litter larger than 1 and 2 . The phenomenon is in agreement with the characteristics of typical nonlinear systems. Moreover, the hysteresis increases with the strength of external excitation. The vibration amplitudes at different frequency ratio 
increase with external excitation amplitudes and frequency ratio range of large amplitude parametric vibration occurrence under weaker external excitation is relatively smaller.

\section{Responses of Stay Cables under Supports' Excitation and Static Wind Load}

The static wind load (in $z$ direction) is applied to the model in Fig. 1 to investigate its effect on parametric vibration. The configuration and natural frequency of the cable is changed under static wind load. The response of $J 34$ under combined supports' excitation and static wind load (basic wind speeds of $50 \mathrm{~m} / \mathrm{s}$ and $100 \mathrm{~m} / \mathrm{s}$ ) are calculated by FEM, and the result at the basic wind speed of $50 \mathrm{~m} / \mathrm{s}$ is shown in Fig. 5. It can be seen that the lateral vibration (in $z$ direction) is raised up due to the static wind load and shows a phenomenon of "flap" in the same frequency of vertical vibration. Further studies indicate that the ratio of vertical displacement and lateral displacement do not change over time. It can be concluded that under combined supports' excitation and static wind load, the cable will vibrate around its deformed curve under self weight and static wind load.

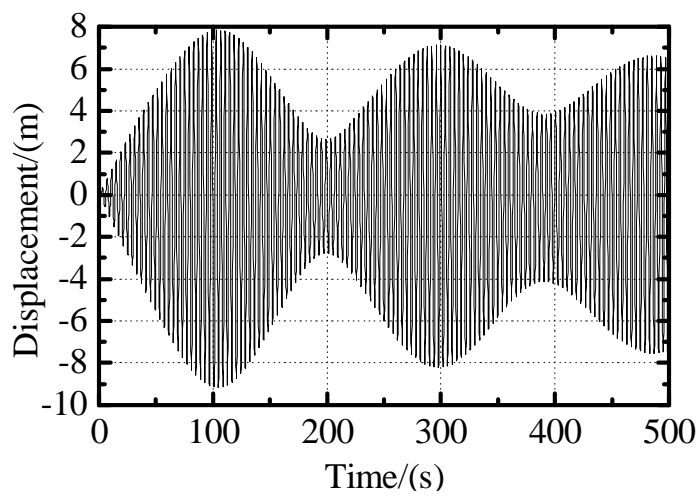

(a) vertical displacement

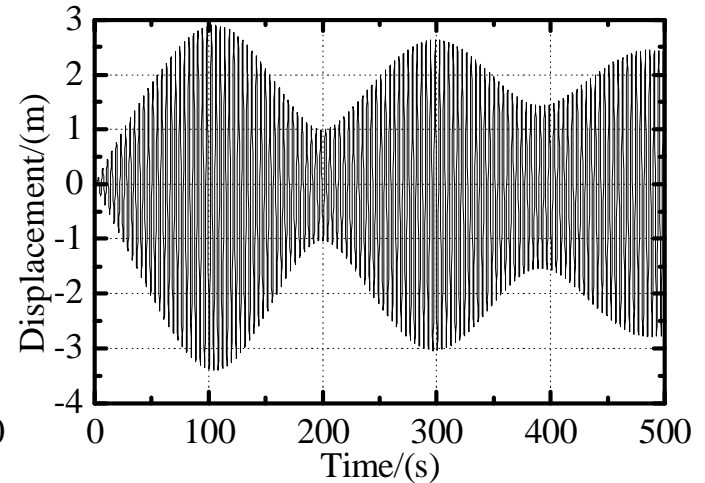

(b) lateral displacement

Fig. 5 Displacement response of $J 34$ at $\xi=0.001, \Omega / \omega_{l}=1, U_{d}=0.0001 L$ and $u=50 \mathrm{~m} / \mathrm{s}$

\section{Conclusions}

The in-plane FF of a stay cable is higher than the out-plane FF, and the difference increases with the offset of its deformed configuration from its initial configuration. The dependence of vibration amplitude on frequency ratio is slightly hysteretic and the hysteresis effect increases with the strength of external excitations. The vibration amplitude at certain frequency ratio increases with the strength of external excitations. The frequency ratio range of large amplitude parametric vibration occurrence increases with the strength of external excitations. Vibrations of stay cables are not perfectly symmetrical about the equilibrium curves and the asymmetry increases with the inclined angles of stay cables. Under combined supports' excitation and static wind load, a stay cable will vibrate around its deformed curve under self weight and static wind load.

\section{Acknowledgements}

This work was supported by the National Program on Key Basic Research Project (973 Program, 2015CB057705, 2015CB057701) and the National Science Foundation of China (51478087).

\section{References}

[1] Yong Xia, Yozo Fujino: Journal of Engineering Mechanics Vol. 132 (2006), p. 279-286

[2] Chen Shuisheng, Sun Bingnan: Chinese Journal of Vibration Engineering Vol. (2) (2002), p. 144-150. (in Chinese) 